Transcontinentales

continentales

Sociétés, idéologies, système mondial

La transition chinoise

\title{
L'origine est aux frontières
}

Espace, histoire et jihad chez les Aït Ba'amran du Sud marocain

\section{Romain Simenel}

\section{CpenEdition}

\section{Journals}

Édition électronique

URL : http://journals.openedition.org/transcontinentales/566

DOI : $10.4000 /$ transcontinentales. 566

ISBN : 978-2-8218-1112-6

ISSN : 1775-397X

Éditeur

Editions de la maison des sciences de l'homme

\section{Édition imprimée}

Date de publication : 31 décembre 2006

Pagination : 129-143

ISBN : 978-2-200-92170-5

ISSN : $1950-1684$

Référence électronique

Romain Simenel, «L'origine est aux frontières », Transcontinentales [En ligne], 3 | 2006, document 8, mis en ligne le 12 octobre 2011, consulté le 08 septembre 2020. URL : http://journals.openedition.org/ transcontinentales/566 ; DOI : https://doi.org/10.4000/transcontinentales.566

Ce document a été généré automatiquement le 8 septembre 2020

Tous droits réservés 


\title{
L'origine est aux frontières
}

\author{
Espace, histoire et jihad chez les Aït Ba'amran du Sud marocain
}

\section{Romain Simenel}

Les travaux de M. Halbwachs sur la mémoire collective ont mis à jour l'existence de rapports étroits entre les représentations collectives de l'espace et la mémorisation du passé. Pour cet auteur, l'espace est modelé de manière spécifique par les différentes sociétés qui « dessinent en quelque sorte leur forme sur le sol» et, ainsi, il devient le support immuable des souvenirs historiques des hommes ${ }^{1}$. L'empreinte particulière qu'un groupe imprime dans l'espace, déterminée en grande partie par son organisation sociale et son mode de vie, jouerait d'une certaine manière, pour ses membres, le rôle d'une carte mémorielle qui guiderait la mémoire de leur passé collectif; comme le résume si bien un historien brésilien, «la mémoire a au moins autant besoin de s'inscrire dans l'espace que de s'inscrire dans le temps ${ }^{2} »$. Cependant, l'approche d'Halbwachs n'explicite pas clairement la distinction qu'elle opère entre mémoire et histoire. Dans son exemple sur la mémoire du lien au sol, ou mémoire juridique, Halbwachs explique que l'espace, empreint de la marque spécifique laissée par le groupe qui l'occupe, permet à chaque instant à la mémoire collective, lorsqu'elle le perçoit, « d'y retrouver le souvenir des droits » et donc l'historique de la propriété ${ }^{3}$. Il raccorde ainsi les souvenirs collectifs inscrits dans l'espace et renvoyant à un passé lointain non vécu par les sujets, qui constituent la mémoire subjectivement collective, à l'histoire réelle de leur communauté territoriale; une histoire qui pour M. Halbwachs est objective, chronologique, " et apparemment indépendante du contexte social dans lequel elle est produite ou consommée ${ }^{4}$ ». Depuis, les recherches en ethnohistoire ont remis en question l'universalisme présupposé de la notion d'histoire telle qu'elle est définie en Occident, en révélant la diversité des manières de la conceptualiser, et le fossé immense qui sépare la façon dont les sociétés mémorisent leur passé et l'histoire objective, si tant est qu'il y en ait une. L'histoire au pluriel est aujourd'hui abordée par les ethnologues comme une construction du passé qui relève d'une mémoire sélective ; le passé ne se façonne pas que dans le souvenir, et l'exemple amazonien a démontré à quel point l'oubli est un pilier de l'histoire ${ }^{5}$. 
Considérant l'espace dans sa dimension historicisante comme nous le propose Halbwachs, la question se pose alors de savoir comment l'espace, tel qu'il est perçu par les hommes, articule la dynamique du souvenir et de l'oubli dans la représentation du passé. Cependant, s'il existe des lieux du souvenir ou des lieux de l'oubli, il existe aussi des lieux oubliés, camouflés ou célébrés. Si la structure spatiale des sociétés influe sur la manière de mémoriser le passé, les représentations du passé participent des perceptions de l'espace. L'homme façonne l'espace en y semant ses souvenirs, souvenirs qui l'amènent à le percevoir autrement. Différemment d'Halbwachs, pour qui l'espace est support de la mémoire du fait de son immuabilité, et qui du coup écarte la question des interactions entre la subjectivité de cette mémoire et celle de l'espace perçu, cet article cherche à mettre l'accent sur la capacité des membres d'une société à transfigurer conjointement l'espace et l'histoire.C'est à un requestionnement de la dialectique entre spatialité et historicité que cet article voudrait contribuer, à partir de l'exemple ethnographique des Aït Ba'amran, confédération tribale du Sud-Ouest marocain, et de l'analyse de la perception particulière que ces derniers ont des frontières de leur territoire politique. Précisons d'entrée que la notion de frontière est ici abordée dans un sens anthropologique plus que dans son acception géopolitique. La frontière territoriale sera avant tout traitée comme un outil de pensée. Comme nous le démontrerons dans cet article, la frontière revêt une certaine épaisseur symbolique et elle est l'objet de représentations tant spatiales qu'historiques.

En allant vers le sud, le territoire de la confédération Aït Ba'amran est la dernière région montagneuse côtière du Maroc avant le Sahara. Les Aït Ba'amran sont en grande majorité berbérophones et sédentaires alors que les tribus plus au sud sont Sahraouis, nomades et arabophones (hassaniya). Néanmoins, parce qu'ils ont été colonisés par les Espagnols, au même titre que les territoires du Sahara occidental, et non par les Français, à la différence des régions des alentours, les Aït Ba'amran ne -cessent de réclamer, pour des raisons politiques plus que culturelles, le rattachement administratif de leur confédération à la province de Guelmim ${ }^{6}$. En vertu de son positionnement stratégique dans l'affaire de la détermination du statut du Sahara occidental, cette province, habitée en grande majorité par des Sahraouis, bénéficie en effet de nombreux avantages économiques et sociaux concédés par le gouvernement marocain. 


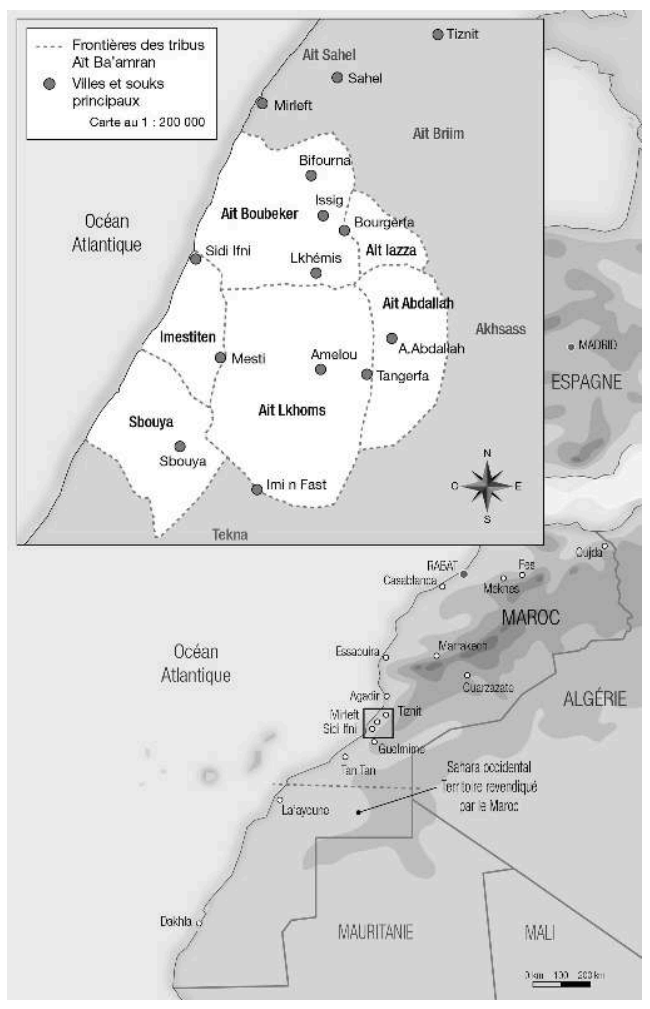

Carte de localisation des Aït Ba'amran

Le territoire politique de la confédération est organisé de manière segmentaire, il est fragmenté en tribus, elles-mêmes composées de fractions qui regroupent des douars habités par des patrilignages. La tribu est une unité politique, "puisqu'elle agit comme un seul corps contre ce qui la menace ${ }^{7} . .$. ». À l'image de la confédération, les tribus ne sont pas organisées traditionnellement selon un pouvoir politique centralisateur ; il n'y a pas un chef mais des grands hommes dont le charisme émerge au gré des alliances entre patrilignages. Le territoire de la confédération et ceux des différentes tribus qui la composent sont clairement délimités dans le paysage, et leurs frontières sont bornées de manière très précise.

Si ces frontières sont locales, elles n'en sont pas moins perçues comme étant au cœur de l'histoire d'une frontière bien plus globale, celle entre le monde musulman et le monde des chrétiens. À ce propos, s'il est question de jihad dans cet article, ce n'est pas dans le sens commun et dualiste d'une guerre religieuse menée par les musulmans contre les impies chrétiens. Bien au contraire, le terme jihad est avant tout employé localement pour désigner une lutte historique contre la colonisation chrétienne à laquelle on donne l'allure d'une guerre sainte fondatrice du territoire. Il ne s'agit jamais de convertir le chrétien, mais bien de le chasser des terres de la confédération. Par ailleurs, le terme jihad est parallèlement compris comme une quête mystique individuelle caractéristique de la démarche historique des saints de la région.

7 Chez les Aït Ba'amran, qui se qualifient eux-mêmes de descendants de bannis, les frontières constituent ainsi la référence cognitive à la représentation de l'altérité et à la construction de l'histoire des origines du territoire. Confins de l'altérité, les frontières sont aussi l'axe de la condensation de l'histoire. Dans ce cadre, les frontières territoriales relèvent tant d'une dimension spatialisante, dans une fonction de 
délimitation des différentes entités territoriales politiques que d'une dimension historicisante, dans un rôle de relais historique entre le territoire et ses origines.

\section{Un territoire sans passé local ?}

8 Territoire fractionné, le pays Aït Ba'amran ne se déploie pas autour d'un centre politique et historique particulier. Si les souks jouent le rôle de centres économiques hebdomadaires, ils ne constituent en rien des centres politiques et encore moins historiques, puisqu'il y est d'usage de ne pas parler du passé ni des anciens, mais de se consacrer au présent, c'est-à-dire au commerce. Le nom de la confédération n'évoque, pour les habitants, aucune référence au passé, et ne suscite étymologiquement aucune interprétation. Le terme «Ba'amran » n'est pas employé pour désigner un ancêtre, un animal totem, ou une humanité exclusive, tout au plus, pour les tribus voisines désignet-il les fils d'un juif appelé Ba'amran. Les noms des tribus qui composent la confédération (Aït Boubeker, Aït Lkhoms, Sbouya, Imestiten...), pourtant composés à partir de noms communs, sont interprétés comme des noms d'ancêtres claniques. Cependant, outre le fait que personne ne revendique de généalogie reliant les hommes d'aujourd'hui à ces ancêtres, ils ne sont l'objet d'aucun récit et ne sont pas considérés comme des fondateurs.

9 Les faits et gestes des amghar, grands hommes d'honneur, encore très influents il y a à peine cinquante ans dans la région et dans tout le $\operatorname{Maroc}^{8}$, n'ont pas été mémorisés et sont totalement absents du discours historique, hormis celui de certains caïds érudits et de quelques personnages très marginalisés comme de vieux poètes. Ils ne sont pas célébrés post mortem, leurs tombes sont comme toutes les autres, et aucun monument ou endroit quelconque ne leur sont associés. Il en va de même du passé guerrier de ces tribus, pacifiées depuis moins de quarante ans et auparavant imbriquées dans des échanges de violences entre groupes laïcs ${ }^{9}$, participant de la structure segmentaire de l'espace social; un passé totalement oublié, même s'il fait l'objet de chroniques et de récits écrits de voyageurs ou d'historiens épiques et héroïques. Une certaine amnésie s'exprime ainsi, dans la société Aït Ba'amran contemporaine, envers tout ce qui relève de l'histoire politique, des conflits passés et des grandes figures charismatiques issues de la région. Plus encore, la structure politique du territoire de la confédération semble ne garder aucune trace de son histoire locale.

10 Les Aït Ba'amran disent de leur pays qu'il est un exil où les bannis et réfugiés politiques originaires de différentes tribus du Maroc ont l'habitude de s'installer, et cela depuis que le roi Salomon en fit une prison. Personne n'a honte d'affirmer son origine extérieure et sa filiation à un ancêtre banni. Dans cette région sèche et aride, les quelques rares sources sont contrôlées depuis très longtemps par ceux que l'on nomme haratin, en arabe, ou issuqin, en berbère, population noire autochtone, distincte des descendants d'esclaves, qui exerce bien souvent les métiers de potier ou de forgeron ${ }^{10}$. Leur présence séculaire est attestée dans de vieux papiers de propriétés et dans les écrits des anciens voyageurs, tel Léon l'Africain ${ }^{11}$. Cependant, le discours local, écrit ou oral, sur l'histoire du pays Aït Ba'amran, alimenté par les fuqaha, savants religieux, ne mentionne jamais l'ancienneté des issuqin. Bien au contraire, leur ancienneté sur le sol est récusée par les autres qui les qualifient de bâtards, de métis descendants d'esclaves et de blancs. Étonnante conception que celle d'une région qui affirme n'avoir aucun autochtone, et n'être ni une terre d'origine ni une terre d'élection, mais une terre de 
destination d'où l'on ne sort pas. Un territoire politique curieusement orphelin de l'histoire, sans origines ni fondations. Dans ce pays d'exil où les hommes se revendiquent d'un ailleurs et nient le passé des autochtones, où l'histoire locale de la confédération est oubliée ou dénigrée, comment se construit donc la mémoire d'une histoire et d'un espace commun? Dans la référence au passé quelle valeur prend le lien au sol?

\section{L'histoire de l'ailleurs aux frontières de l'ici}

Le discours commun à l'ensemble des Aït Ba'amran sur le passé de leur pays porte essentiellement sur les saints. Comme toutes les tribus du Maroc, les Aït Ba'amran ont leurs saints. Figure du passé puisqu'il n'en existe pas de vivant de nos jours, le saint est considéré comme un homme de religion porteur de la baraka (bénédiction divine) ${ }^{12}$ jouant le rôle d'intercesseur entre Dieu et les hommes. Les Aït Ba'amran cultivent le souvenir des saints au travers des nombreuses visites rituelles aux endroits qui leur sont associés.

Les saints sont matérialisés dans l'espace par le biais de mausolées, de simples chambres funéraires, de cimetières ou de tombes éparses entourées d'un cercle en pierres où ils sont censés être enterrés, des zawiya dont ils seraient les fondateurs, des puits qu'ils auraient creusés, des cairns (monticules de cailloux allant de trente centimètres à un mètre cinquante) qu'ils auraient construits, et enfin des arganiers massifs plantés de leurs mains, ou sous l'ombrage desquels ils auraient trouvé repos. Tous les saints connus à l'échelle de la confédération sont localisés le long des frontières des territoires politiques des tribus et fractions qui la composent. Les mausolées et les cairns de saints blanchis à la chaux, ainsi que les arganiers géants qui portent leurs noms, sont, dans le paysage, les marqueurs les plus visibles des limites territoriales des tribus. Chaque catégorie de saint a sa spécificité géographique : si les mausolées ou cercles en pierres des saints Ida ou Simelel ${ }^{13}$ trônent sur les plus hauts sommets qui délimitent les frontières ou s'enracinent aux bords des voies de passage les traversant, les mausolées des saints Ouled bou Sbah ${ }^{14}$ ou Rrgraga ${ }^{15}$ bordent les principales criques accostables des côtes. Quant aux saints les plus célèbres, leur présence est systématique aux nœuds stratégiques des frontières des tribus et fractions. Pour le Haut Atlas, Ernest Gellner avait déjà relevé la présence récurrente des saints aux frontières des tribus ${ }^{16}$, fait qu'il interpréta dans le cadre de son analyse de la structure segmentaire. Comme chez les Ait Ba'amran, les marques de saints sont disposées sur le fil des frontières aux endroits stratégiques constituant autant de coordonnées qui maillent le territoire et l'organisent, tant du point de vue de la structure politique que de celui de la mobilité des hommes. Pour E. Gellner, l'espace d'élection d'un saint est « dans le périlleux territoire entre deux groupements tribaux majeurs en état d'hostilité latente ou réelle ${ }^{17} \%$. La frontière occupée par les saints devient un espace de médiation, permettant de gérer les conflits tribaux et d'assurer le passage du commerce, et non pas une limite territoriale hermétique. Néanmoins, la fonction politique de l'implantation des saints aux frontières semble être ignorée dans la vision historique qu'en ont aujourd'hui les habitants. L'aspect pacificateur du saint et son rôle d'intermédiaire entre les tribus sont des réalités historiques qui ne sont pas reproduites dans le discours sur le passé, les anciens conflits tribaux étant désormais oubliés. Dans le vécu quotidien de l'espace, les sites saints situés aux frontières sont 
cependant bien des points de passage, des étapes qui balisent l'itinéraire de marche des hommes qui s'y arrêtent pour un instant ou pour une nuit, et repartent vers leurs destinations, non sans avoir préalablement psalmodié une prière et célébré le saint par un "sadaka fissa bil'ilah ", " une aumône sur le chemin de Dieu ». Chaque traversée des frontières prend l'allure d'une visite rituelle éveillant le souvenir des saints dans la cadence du corps.

Les faits et gestes des saints des frontières territoriales sont soigneusement racontés au travers d'une abondance de récits oraux, sous la forme de légendes, de poèmes et d'anecdotes que chacun connait. Ils relatent avant tout comment ils sont arrivés, la manière dont ils se sont installés et les miracles qu'ils réalisèrent en ces terres. Car le saint est toujours de l'extérieur. Conduit par sa chamelle ou guidé par son bâton, le saint incarne l'errance qui ne s'arrête que par l'intermédiaire de sa baraka. Le saint tisse un lien entre le territoire où il finit par s'enraciner et ses origines extérieures. L'espace frontalier attribué à un saint et à ses éventuels descendants -n'appartient ni à l'une ni à l'autre des tribus qu'il sépare, il est apparenté au territoire d'origine du saint. Les frontières des tribus Aït Ba'amran sont ainsi parsemées d'espaces saints affiliés à de nombreuses régions du Maghreb et d'Orient, comme le Tafilalt, Ouazzane ou la région de Tafraoute pour ce qui est du Maroc, mais aussi la Tunisie, l'Égypte ou encore l'Éthiopie. Toutes ces régions ou pays sont perçus comme des grands centres du savoir musulman et sont le théâtre de nombreuses légendes. Les espaces saints représentent, dans le cosmos, le tiers vide du Monde (tlt $n$ khaliya), les deux autres étant constitués du tiers de mers et du tiers de déserts occupés par les géants Gog et Magog. Retraite pour les saints et les hommes, ces sites sont aussi des espaces de communication avec les génies, appelés jnoun ou ljoad. Par l'intermédiaire de la figure des saints, l'espace local est délimité par un ailleurs mythifié qui s'intercale entre les tribus voisines anciennement ennemies. Ce qui délimite le territoire de telle tribu par rapport à celui de telle autre, faisant pourtant partie de la même confédération, est un espace teinté d'un ailleurs bien plus exotique que le territoire voisin. C'est un peu comme si toutes les ambassades à Paris étaient alignées le long des frontières des arrondissements et que, pour passer de l'un à l'autre, il fallait traverser un espace affilié à un pays étranger. Or, cet ailleurs qui définit les degrés de l'ici se trouve être le prisme d'une mémoire historique commune incarnée par la figure du saint. Paradoxalement, l'histoire du pays Aït Ba'amran telle qu'elle transparaît au travers de l'itinéraire des saints est avant tout une histoire de l'ailleurs dont le souvenir s'enracine aux frontières du local. Les saints localisés aux frontières témoignent d'une historicité, perceptible dans le paysage, qui s'inscrit entre l'ici et l'ailleurs.

\section{Le souvenir des chrétiens sur les ruines du passé}

14 À proximité des sites saints frontaliers, les habitants localisent toujours les traces d'une ancienne présence chrétienne (rûmi). Des ruines, telles que celles de greniers à grains ou d'habitations, de bergeries, d'anciens fours à chaux ou de silos souterrains sont attribuées à d'anciens chrétiens. Plusieurs catégories de chrétiens sont associées à ces ruines. Les brtgz ${ }^{18}$, qui sont considérés comme les plus anciens, les spagnol, les ingliz et les francaoui.

D'un point de vue archéologique et historique, ces ruines, mis à part les rares vestiges de la colonisation espagnole de ce siècle, ne sont pas d'origine chrétienne, mais bien 
l'œuvre d'anciens habitants du pays. Les Portugais, les Espagnols et même quelques armateurs anglais aventuriers ont bien fréquenté les côtes Aït Ba'amran, et cela dès le $\mathrm{XV}$ s siècle, pour des raisons commerciales. Les Espagnols ont même construit, dès le XVII siècle, un comptoir connu sous le nom de Santa Cruz de Mar Pequenia, probablement au sud du pays Aït Ba'amran, puis ils ont occupé la ville de Sidi Ifni jusqu'en 1969. Cependant, ni les uns ni les autres ne sont à l'origine de ces ruines. Les archéologues de la région ${ }^{19}$ affirment à ce propos qu'ils n'ont retrouvé à ce jour aucune trace de la présence portugaise sur la côte Aït Ba'amran, ni dans les terres. Cette association des chrétiens avec des traces du passé de la culture locale se retrouve un peu partout au Maroc. Dans le Haut Atlas, sur le plateau du Yagour, tout comme dans la région de Tafraoute, sur les bords de l'oued Tamanart, des gravures rupestres du paléolithique sont désignées comme l'œuvre des brtgz. L'incongruité de ces énigmes portugaises ou chrétiennes a attiré la curiosité de nombreux érudits en histoire et archéologie parmi les officiers français en poste au Maroc. Ces derniers menèrent des enquêtes pour relever et authentifier les sites témoignant soi-disant d'une implantation ancienne des Portugais dans les terres. Déçus, tous conclurent bien sûr à l'origine berbère des ruines qu'on leur avait présentées comme chrétiennes ${ }^{20}$.

Les ruines attribuées aux chrétiens, perchées aux sommets des montagnes, épousant la courbe des cols ou nichées dans les estuaires des principaux oueds, ont toutes la caractéristique spatiale d'être à proximité d'un site saint frontalier et de longer les abords des frontières territoriales de la confédération, des tribus ou des fractions. Les ruines situées au cœur des territoires sont assez rares et ne sont jamais affiliées aux chrétiens, excepté celles ancrées sur le territoire des issuqin (population noire autochtone). Contrairement aux sites saints qui leur sont adjacents, l'espace sur lequel s'enracine ces ruines est collectif et est utilisé comme zone de pâturage par les tribus. La présence de constructions à usage collectif à proximité des saints rappelle l'explication donnée par E. Gellner sur le rôle de protecteur des saints envers les infrastructures collectives en temps de guerre ${ }^{21}$. Cette présence d'habitations fortifiées, de greniers ou autres édifices collectifs sur les hauteurs des frontières est liée à une ancienne manière d'occuper le territoire caractérisée par deux temps d'habitations : un temps de paix, et un temps de guerre et de razzias intertribales appelé siba. Si le temps de paix est matérialisé dans le territoire par des habitations dans les plaines, au centre de l'espace cultivable, celui de la guerre est inscrit aux sommets des plus hauts massifs, là où s'accrochent des greniers à grains où l'on cachait les récoltes et là où sont camouflés des bastions villageois, refuges lors des attaques. En période de razzias, les habitants quittaient leurs résidences habituelles pour aller vivre en haut des montagnes, près des greniers collectifs à proximité des saints. De ce fait, les terroirs ont été occupés de manière alternée, tant de l'intérieur vers leurs périphéries que de leurs périphéries vers l'intérieur.

Toutes ces ruines dispersées le long des frontières, vestiges des périodes de siba, sont perçues aujourd'hui comme d'anciennes infrastructures militaires des chrétiens colonisateurs. Les silos souterrains sont assimilés à des caches d'armes, les greniers à grains qui épousent la forme des socles rocheux auxquels ils s'accrochent, à des prisons, les ruines de bergerie à d'anciennes tranchées. Quant aux fours creusés dans le sol, utilisés localement et depuis des siècles pour cuire la chaux nécessaire au blanchiment des murs des mausolées et d'autres édifices religieux, tous auraient été construits par les chrétiens qui avaient, dit-on, l'habitude de chauler leurs propres édifices militaires. Les territoires des tribus sont ainsi encerclés par le souvenir de la 
présence de militaires chrétiens. Suivant la démarche de $\mathrm{M}$. Halbwachs, selon laquelle l'espace est un support immuable pour la mémoire collective, les ruines d'édifices collectifs localisées sur les frontières devraient jouer le rôle de bornes spatiotemporelles à partir desquelles se construit le souvenir collectif du temps des razzias. Or, si ces ruines sont bien le support d'une mémorisation du passé, l'image qu'en ont les habitants - à savoir les traces d'une ancienne présence militaire chrétienne - diffère radicalement de celle que l'on attendrait a priori, c'est-à-dire les restes d'une ancienne organisation spatiale fondée sur la défense. Reste à savoir ce que la perception particulière que les Aït Ba'amran ont des ruines frontalières révèle de leur vision d'un passé continuellement tournée vers l'extérieur. Dans l'espace vécu des Aït Ba'amran, l'assimilation de ruines à des infrastructures militaires chrétiennes conduit à percevoir les frontières territoriales des tribus et fractions comme d'anciennes zones de conflits contre les chrétiens, figure de l'altérité.

\section{L'espace du jihad et l'oubli des guerres locales} délimitation des frontières tribales, mais elles sont aussi des marqueurs d'événements. Les traces de chrétiens sont toujours associées à des événements relatifs au jihad, mettant en scène des sièges héroïques contre des places fortes ennemies. L'un de ces événements relatés serait à l'origine du nom de Sidi Ifni, ville principale de la confédération située en bord de mer et dont le territoire est surplombé par la montagne Boulalam. La ville aurait été occupée jadis par des brtgz : ils auraient dressé leur drapeau au sommet de la montagne et auraient suspendu sur ses pentes des lampes à huile pour surveiller les moudjahidin Aït Ba'amran. Trois cent cinquante d'entre eux se seraient cachés au pied de la montagne et, de là, auraient détruit les lampes puis envahi Ifni et massacré ses habitants. D'après les fuqaha, savants religieux, le nom Ifni, dérivé du terme arabe classique ifna (il est mort) qui signifie localement " endroit dévasté », viendrait de cet épisode. Des ruines de bergeries et de greniers à grains au sommet de la montagne sont désignées comme les dernières traces de la présence des brtgz.

Pour être un lieu de mémoire, le lieu doit être aussi un lieu d'oubli ; le souvenir des lieux de batailles imaginaires contre les chrétiens contraste avec l'oubli des conflits intertribaux qui s'y sont réellement déroulés. Ainsi en est-il du lieu dit dfayer siba (les nattes de l'anarchie), plaine située aux frontières tribales où se déroulèrent de nombreux combats internes à la confédération, et qui est aujourd'hui décrit comme ayant été le théâtre d'un bombardement français au début du siècle.

Certains des saints postés aux frontières sont clairement associés au jihad et, bien souvent, des cimetières de martyrs jouxtent leurs tombeaux. Certaines de ces figures du jihad sont reconnues comme étant anciennes, tels les saints Ida ou Simelel pour ce qui est de l'intérieur des terres, les Ouled bou Sbah ou les Rrgraga pour la côte; d'autres sont considérées comme plus récentes, tels les martyrs de 1957 morts contre les Espagnols. Néanmoins, à propos de ces saints et martyrs, la réalité historique diverge bien souvent de la représentation qu'en ont les habitants. Les saints Ida ou Simelel étaient de simples savants religieux et non des meneurs de jihad. Les Aït Iazzawihda, dont les cimetières jonchent les frontières en plaine, étaient des guerriers à la solde de la zawiya d'Assa, dont la mission était plus de combattre les hérésies locales que les 
chrétiens ${ }^{22}$. Les cimetières dénommés Sidi Hucein, champs de pierres anonymes, rassemblent les tombes d'élèves d'un grand savant religieux originaire de Tamgrout du nom de Sidi Hucein, adepte de la confrérie Nassiriya, et non celles de jeunes martyrs embrigadés contre les chrétiens par un meneur de jihad. Quant au saint Sidi Ali Ifni, dont le mausolée en bord de mer marque l'entrée de la ville qui porte son nom, il fut simplement, dit-on, chargé par le Sultan du Maroc de surveiller la côte contre l'intrusion d'espions chrétiens. Enfin, les chorfa, lignages reconnus descendants de saints et du prophète Mohammed, qui occupent justement certains sites saints frontaliers, affirment que leurs ancêtres étaient de vrais leaders du jihad contre les chrétiens alors que leur rôle consistait plutôt à pacifier les conflits tribaux internes à la confédération.

21 Aux frontières, la contiguïté et la complémentarité entre les sites saints et chrétiens, entre un ailleurs porteur des valeurs de l'islam et une altérité stigmatisée sur la figure du chrétien, plantent le décor du souvenir d'un jihad qui inonde la mémoire historique des Aït Ba'amran. La perception des frontières établit ainsi une jonction symbolique entre les tensions du temps et de l'espace. L'association, sur les frontières territoriales, entre les marques de saints et les traces de chrétiens vient substituer au passé des guerres tribales celui d'un jihad contre les chrétiens. Les anciennes querelles entre tribus, ainsi que le rôle pacificateur des saints, sont camouflés par la référence au jihad. Dans le souvenir des Aït Ba'amran, une guerre en a remplacé une autre, une altérité en a remplacé une autre, l'ennemi n'est plus la tribu voisine mais le chrétien, et c'est de la sorte qu'ils perçoivent leur espace commun.

\section{Le jihad à l'origine du territoire}

La présence des saints et des chrétiens aux frontières est explicitée au travers d'un récit mythologique sur l'occupation du sol en pays Aït Ba'amran, produit très probablement par les savants religieux (fuqaha) et les étudiants coraniques ( $t l b a$ ) de la région, puis véhiculé par tous.

Les premiers musulmans arrivés dans ce bled et l'ayant découvert sont des frères saints d'origine de la région de Tafraoute. Avant leur arrivée, le pays était recouvert par la mer, excepté certaines localités aujourd'hui situées dans les terres et aux frontières, mais jadis côtières et sous la forme d'îlots émergés constitués de criques servant de ports naturels (aftass). Ces derniers étaient occupés par les brtgz, des chrétiens pêcheurs de fruits de mer et de poissons de qualité comme le bar. Quand les saints sont arrivés, quelqu'un que l'on nomme Abouraz, certains disent qu'il s'agit de Duul Qarnain (Alexandre le Grand), a ouvert Bouraz (détroit de Gibraltar) et l'eau de la mer s'est écoulée de l'autre côté. Le niveau de la mer a diminué sur la côte océanique marocaine laissant à découvert les plaines et collines du pays Aït Ba'amran. C'est alors que les saints et les moudjahidin se sont battus avec les brtgz, armés de bâtons crochetés (alkassou), de pierres et de lance-pierres (ildi, tizina), car à l'époque il n'y avait pas de fusils, jusqu'à ce qu'ils les aient repoussés au large. Ce pays est resté comme ça, et après, ceux qui sont venus ont pu s'y installer.

Dans ce récit, les brtgz sont présentés comme les autochtones du pays Aït Ba'amran ; ils étaient là avant puisque les saints les y ont trouvés. Gellner avait déjà souligné que «la croyance que les proto-habitants de la région étaient des Portugais (Portkiz, Portikzi) troglodytes est répandue [...] partout dans l'Atlas et dans les autres régions berbères du Maroc ${ }^{23}$ ». Cependant, le mythe ne les donne pas comme les premiers occupants de l'espace dans sa totalité, puisque leur occupation des terres se limite aux sommets 
émergés, aujourd'hui marqueurs des frontières. Les brtgz ne sont que les autochtones des frontières, ils incarnent un passé qui ne concerne que celles-ci. De surcroît, les brtgz sont des pêcheurs, ils n'ont donc pas entrepris d'exploitation agraire et n'ont fait aucune utilisation du sol. Les frères saints, d'origine extérieure mais provenant d'une région berbère, sont mis en scène en tant que meneurs du jihad, garants de l'existence territoriale du pays Aït Ba'amran et premiers gestionnaires du sol, puisque leur arrivée coïncide avec le retrait de la mer. Le mythe arrange l'histoire, avec l'entremise du prophète Alexandre le Grand ${ }^{24}$, pour que la terre fertile des plaines et des collines soit vierge lors de l'arrivée des saints. Par l'intermédiaire de ces derniers, l'avènement de l'islam en pays Aït Ba'amran coïncide avec la possibilité offerte aux futurs arrivants musulmans de cultiver la terre, mettant un terme à un temps chrétien où seule la pêche était pratiquée. Ce rôle de premier gestionnaire du sol est d'ailleurs constamment relayé par l'hagiographie des saints qui leur attribue l'origine des sources d'eau du pays.

Ce discours mythologique apparait avant tout comme un camouflage de l'autochtonie et de l'ancienneté d'occupation du sol puisque tout ce qui est antérieur aux saints du jihad est brtgz, donc chrétien; le passé de la terre se calque de la sorte sur celui de l'islam. Cette tendance à dissimuler une ancienne occupation du sol se retrouve aussi dans les Aurès où l'on remarque « quelque chose du refus ou du malaise des montagnards envers l'autochtonie ${ }^{25}$ ». L'histoire du pays Aït Ba'amran en tant que terre d'islam commence avec les saints du jihad. Si l'arrivée des premiers saints entrâne le départ des brtgz, il ne signifie pas la fin du temps du jihad ni la disparition des chrétiens, qui, d'après ce que nous en disent les mythes et l'interprétation des ruines, semblent revenir de manière continue sous la forme, cette fois, de spagnol, d'engliz et de françaoui. De même, à chaque nouvelle vague de chrétiens correspondent de nouvelles arrivées de saints assurant la perpétuation du jihad. Par-delà les mausolées, les ruines et les mythes, le jihad est donné à voir comme principe d'appropriation terrienne aux dépens de l'autochtonie et de l'ancienneté -d'occupation du sol; le droit de conquête prend le pas sur le droit du sol selon une dialectique islam/rûmi (islam/chrétiens) imprimée aux frontières du territoire.

\section{D'hier à jadis : les frontières du jihad}

Pour ce qui est de l'histoire contemporaine, en 1917 puis en 1957, une armée de résistance locale s'est réellement soulevée, d'abord contre les colonisateurs français puis contre les Espagnols. En 1957, la révolte des Aït Ba'amran dura huit mois et aboutit à un accord passé entre les autorités espagnoles et les Aït Ba'amran, qui limitait l'occupation espagnole à la seule ville de Sidi Ifni. Exemple unique au Maghreb d'une tribu réussissant à libérer d'elle-même et de manière définitive son territoire des colonisateurs, cet exploit fut très rapidement convoité par le mouvement nationaliste, qui se le réappropria comme symbole de l'histoire contemporaine du Maroc. Plus de 6000 cartes de résistants furent distribuées aux Aït Ba'amran, et le nom de la confédération est aujourd'hui cité dans les livres scolaires d'histoire. Soulignons toutefois que les événements majeurs de cette résistance contemporaine contre la colonisation, qualifiée de jihad, par les plus grands hasards de l'«histoire » se sont déroulés, de mémoire d'habitants ou d'historiens, aux points les plus stratégiques des frontières des tribus et confédérations de la région, marqués par le sceau des saints les plus célèbres. 
Sidi Mohammed ben Abdallah est un des saints côtiers les plus connu de tout le pays Aït Ba'amran. Il serait mort en 1146 de l'hégire (1733 apr. J-C). On raconte de lui qu'il était un savant soufi originaire du Tafilalt mais qu'il aurait grandi et vécu en Tunisie. Il voyagea partout dans le Maghreb puis s'installa sur la côte Aït Ba'amran. Son bâton, qui le guida le long de son itinéraire, lui indiqua l'emplacement de sa retraite, là où se trouve actuellement son mausolée. Sidi Mohammed ben Abdallah serait arrivé là juste après la fuite des brtgz, chassés par un jihad mené par Sidi Ahmed ou Moussa, saint renommé de la région du Tazerwalt, et juste avant l'intrusion des Espagnols. Le discours oral témoigne du rôle du saint dans la construction spatiale du territoire des Aït Ba'amran en racontant comment il aurait emmené avec lui vingt-deux couples de jnoun, les répartissant le long des frontières des tribus de la région. Le site saint de Sidi Mohammed ben Abdallah est niché au cœur d'une large crique, dans l'embouchure d'un oued faisant office de frontière entre deux tribus des Aït Ba'amran, celle des Aït Mirleft et celle des Aït Nss. Cet oued servait aussi d'ancienne frontière entre la partie colonisée par l'Espagne et le territoire colonisé par la France. De part et d'autre des deux rives de l'embouchure de l'oued Salgumad, les ruines de postes douaniers espagnols et français posés en regard sont appréhendées comme les témoins de cette époque récente de la colonisation. En 1957, une bataille aurait éclaté à Sidi Mohammed ben Abdallah avec la prise d'assaut, par un groupe de combattants Ait Ba'amran, du poste de douane espagnol. Les échanges de tirs firent plusieurs morts des deux côtés. Le souvenir de cet événement est matérialisé par la présence, à côté du mausolée, d'un cimetière de martyrs à l'entrée duquel on trouve une plaque commémorant les faits et gestes de ces hommes morts pour l'indépendance. Chaque année, au mois de février, un rassemblement rituel autour du saint est organisé pendant trois jours par les officiants religieux. C'est alors l'occasion de saluer ${ }^{26}$ le saint et les martyrs, ce que font de nombreux habitants de la région mais aussi les autorités locales et les représentants des ministères marocains qui se rassemblent le deuxième jour dans une prière collective des moudjahidin (salat lmujahidin).

Au sein du cimetière, à côté des tombes des martyrs de 1957, seraient enterrés d'autres martyrs d'une époque plus ancienne, celle de la colonisation brtgz qui prit fin avec le jihad du grand saint du Tazerwalt. Pour les Aït Ba'amran, le souvenir de ces brtgz est perceptible dans le paysage. L'oued dans lequel se niche le site saint porte le double nom de "oued Sidi Mohammed ben Abdallah » et de "oued Salgumad». Les habitants de la région sont fiers d'expliquer que le terme "Salgumad», qui veut aussi dire serpent dans le parler courant, dérive de "Samtmat ", nom d'un ancien général brtgz qui gérait le port d'où partaient les produits de la pêche ainsi que d'autres richesses de la région. Les restes de mortier sur la plage sont désignés comme étant les vestiges $\mathrm{du}$ port brtgz, et l'un des murs de la mosquée adjacente au mausolée aurait été construit sur la ruine d'un ancien mur d'un comptoir brtgz. Cependant, le jihad ne semble pas avoir commencé uniquement avec les brtgz. Le site porte aussi la trace des gestes héroïques du prophète Ali, gendre du prophète Mohammed, qui d'après les légendes locales, aurait été pourchassé par les chrétiens depuis le Mashreq (l'Orient) jusqu'au site de Sidi Mohammed ben Abdallah, en pays Aït Ba'amran. Là, il aurait trompé les chrétiens avec l'aide de Dieu, qui lui aurait ouvert un passage dans le monticule rocheux situé au milieu de la plage, et lui aurait permis d'entrer dans la mer avec son cheval pour en ressortir de l'autre côté de la crique par une arche miraculeusement apparue que l'on nomme "la cuisine de Moulay Ali » (Anual Moulay Ali). Une pierre près de l'arche porterait encore la marque du sabot de sa jument tout juste sortie de la 
mer. Les traces du passage d'Ali placent le site dans l'histoire des origines de l'islam, même si Ali n'est jamais venu au Maroc et n'y a pas combattu de chrétiens. Néanmoins, le combat d'Ali résonne en écho à celui de Sidi Ahmed ou Moussa contre les brtgz ou à celui des moudjahidin de 1957 contre les Espagnols, alimentant le souvenir d'un jihad universel et intemporel en ces lieux. Dans un même site sont ainsi rassemblés les marqueurs de différentes époques de la colonisation et du jihad. Mais de tous ces fragments du passé contenus dans un seul endroit, il est impossible de dresser une chronologie logique. L'épisode d'Ali n'est ni avant, ni après l'épisode de Samtmat, le Portugais autochtone, puisqu'il se situe dans des temporalités différentes, il est tout simplement à côté. Pour les Aït Ba'amran, ces événements ne se succèdent pas dans le temps mais bien dans l'espace. Sur le site de Sidi Mohammed ben Abdallah, c'est toute l'histoire du jihad qui se déroule de lieux en lieux.

D'autres sites saints frontaliers du pays Aït Ba'amran font la jonction spatio-temporelle entre différentes époques de la colonisation, entre des événements récents et réels de la lutte contre l'occupation espagnole et française et des événements légendaires d'un jihad originel. Ces sites ont en commun de s'intercaler sur les nœuds des frontières, d'être marqués du sceau des saints, des martyrs et des chrétiens, et d'être perçus comme le théâtre de hauts faits du jihad.

Tous ces lieux-dits égrainés le long des frontières territoriales tissent une continuité dans l'espace et le temps entre les anciens saints du jihad et les martyrs de 1957, entre les brtgz et les colons espagnols d'il y a trente-cinq ans. Ces traces de chrétiens et ces marques de saints du jihad et de martyrs mettent ainsi en scène la frontière comme un espace de jihad suggérant une colonisation permanente du pays Ait Ba'amran et une guerre sainte continue. C'est comme si le temps s'était arrêté aux frontières, cristallisant le souvenir du passé sur le thème du jihad. Les paysages frontaliers sont perçus selon une logique d'affrontement contre les colonisateurs de toutes époques, théâtre de l'avènement sans cesse renouvelé de la culture musulmane émergeant d'un temps chrétien. Pour ces exilés des origines, ainsi que se définissent les Ait Ba'amran, la frontière territoriale et politique revêt un sens historicisant selon lequel les événements du passé se déroulent exclusivement de l'extérieur vers l'intérieur, comme si l'histoire locale provenait systématiquement d'ailleurs.

\section{BIBLIOGRAPHIE}

- BLoch Maurice, « Mémoire autobiographique et mémoire historique du passé éloigné », Enquête, $\mathrm{n}^{\circ} 2,1996$, p. 59-76

- CiARCIA Gaetano, « Notes autour de la mémoire dans les lieux ethnographiques », Ethnologies comparées, $\mathrm{n}^{\circ}$ 4, 2002, p. 1-17, http://alor.univ-montp3.fr/cerce/revue.htm.

- Colonna Fanny, « Ce que les paysans disent de leurs ruines. Aurès, les années soixante-dix », Bulletin économique et social du Maroc, $\mathrm{n}^{\circ}$ 159-160-161, 1987, p. 249-267 
- FARIAS Paolo Fernando de Moréas, « Histoire contre Mémoire : épigraphie, chroniques, tradition orale et lieux d'Oubli dans le Sahel Malien », série Conférences de la Chaire du patrimoine MarocoAfricain, Rabat, 1993.

- GELLNER Ernest, Les saints de l'Atlas, Saint-Denis, Bouchène, 2003 (réédition).

- HallBWAChs Maurice, La mémoire collective, Paris, PUF, 1968.

- JAMous Raymond, Honneur et Baraka. Les structures sociales traditionnelles dans le Rif, Paris et Cambridge, Éditions de la Maison des sciences de l'homme et Cambridge University Press, 2002 [1981].

- MAIтRот Capitaine, « Les ruines dites portugaises des Doukkala », Archives berbères, vol. 1, fasc. 4, 1916, p. 279-292.

- NoRA Pierre (dir.), Les lieux de mémoire, 3 tomes, 7 volumes, Paris, Gallimard, 1984-1993.

- TAYLOR Anne-Christine, «L'oubli des morts et la mémoire des meurtres », Terrain, n² 28, 1997, p. 83-96.

\section{NOTES}

1 - M. Halbwachs, La mémoire collective, Paris, PUF, 1968, p. 166.

2 - P.F. M. Farias, «Histoire contre Mémoire : épigraphie, chroniques, tradition orale et lieux d'oubli dans le Sahel malien", série Conférences de la Chaire du patrimoine maroco-africain, Rabat, 1993, p. 7.

3 - M. Halbwachs, op. cit., p. 149.

4 - M. Bloch, "Mémoire autobiographique et mémoire historique du passé éloigné ", Enquête, $\mathrm{n}^{\circ}$ 2, 1996, p. 63. 5 - A.-C. Taylor, «L'oubli des morts et la mémoire des meurtres », Terrain, $\mathrm{n}^{\circ} 28$, p. 83.

6 - L'été dernier, une manifestation a été organisée par les habitants de Sidi Ifni pour réclamer à l'État marocain, dans un premier temps, trois actions concrètes : la reconstruction du port fondé par les Espagnols, la construction de la route reliant Sidi Ifni au Sahara (Tan-Tan), et leur rattachement à la province de Guelmin. La manifestation a eu comme conséquence première, l'arrestation de plusieurs centaines de personnes.

7 - R. Jamous, Honneur et baraka. Les structures sociales traditionnelles dans le Rif, Paris et Cambridge, Éditions de la maison des sciences de l'homme et Cambridge University Press, 2002, p. 33.

8 - Voir R. Jamous, op. cit., p. 100-122

9 - La société Aït Ba'amran est composée de quatre catégories sociales, les i'amin (laïcs, jadis guerriers), les chorfa (reconnus descendants du prophète Mohammed), les issmgn (descendants d'esclaves) et les issuqin (population noire qualifiée de métisse).

10 - Pour plus d'informations sur les haratin dans le Sud marocain, voir les publications de M. Naimi, et notamment le chapitre 1 de l'ouvrage La dynamique des alliances ouest-sahariennes, Paris, Éditions de la maison des sciences de l'homme, 2004.

11 - Léon l'Africain, De l'Afrique, Paris, Cordier, 1830.

12 - Voir R. Jamous, op. cit.

13 - Tribu de la région de Tafraoute.

14 - Tribu d'origine saharienne.

15 - Tribu de la région d'Essaouira.

16 - E. Gellner, Les saints de l'Atlas, Saint-Denis, Bouchène, 2003 (rééd.), p. 135-136, 144, 154, 235, 255,260 et 287. 
17 - E. Gellner, op. cit., p. 144.

18 - Terme sans nul doute dérivé de Bortugal (Portugal), même s'il est rare qu'il renvoie consciemment à l'État du Portugal. À ce propos, un jour, un de mes interlocuteurs me posa la question de savoir si les brtgz étaient des engliz!

19 - Les archéologues de la délégation du patrimoine culturel de la région de Tiznit.

20 - Capitaine Maitrot, «Les ruines dites portugaises des Doukkala », Archives berbères, vol. 1, fasc. 4, 1916, p. 279-292.

21 - E. Gellner, op. cit., p. 255.

22 - À ce sujet voir V. Monteil, "Chronique de la zaouia d'Assa ", in Mélanges Mohamed El-Fassi, Rabat, Université Mohamed-V, 1967, p. 81-90.

23 - Op. cit., p. 171.

24 - Dans la tradition coranique, Alexandre le Grand, appelé Seydna Duul Qarnain, est considéré comme un prophète.

25 - F. Colonna, "Ce que les paysans disent de leurs ruines. Aurès, les années soixante-dix », Bulletin économique et social du Maroc, $\mathrm{n}^{\circ}$ 159-160-161, 1987, p. 249.

26 - Traduction du terme tslim, qui vient de l'arabe salama, la paix, employé pour désigner l'acte de visiter et de prier le saint.

1. M. Halbwachs, La mémoire collective, Paris, PUF, 1968, p. 166.

2. P. F. M. Farias, «Histoire contre Mémoire : épigraphie, chroniques, tradition orale et lieux d'oubli dans le Sahel malien", série Conférences de la Chaire du patrimoine maroco-africain, Rabat, 1993, p. 7.

3. M. Halbwachs, op. cit., p. 149.

4. M. Bloch, « Mémoire autobiographique et mémoire historique du passé éloigné », Enquête, $\mathrm{n}^{\circ} 2$, 1996, p. 63.

5. A.-C. Taylor, « L'oubli des morts et la mémoire des meurtres », Terrain, $n^{\circ} 28, p .83$.

6. L'été dernier, une manifestation a été organisée par les habitants de Sidi Ifni pour réclamer à l'État marocain, dans un premier temps, trois actions concrètes : la reconstruction du port fondé par les Espagnols, la construction de la route reliant Sidi Ifni au Sahara (Tan-Tan), et leur rattachement à la province de Guelmin. La manifestation a eu comme conséquence première, l'arrestation de plusieurs centaines de personnes.

7. R. Jamous, Honneur et baraka. Les structures sociales traditionnelles dans le Rif, Paris et Cambridge, Éditions de la maison des sciences de l'homme et Cambridge University Press, 2002, p. 33.

8. Voir R. Jamous, op. cit., p. 100-122

9. La société Aït Ba'amran est composée de quatre catégories sociales, les i'amin (laïcs, jadis guerriers), les chorfa (reconnus descendants du prophète Mohammed), les issmgn (descendants d'esclaves) et les issuqin (population noire qualifiée de métisse).

10. Pour plus d'informations sur les haratin dans le Sud marocain, voir les publications de M. Naimi, et notamment le chapitre 1 de l'ouvrage La dynamique des alliances ouest-sahariennes, Paris, Éditions de la maison des sciences de l'homme, 2004.

11. Léon l'Africain, De l'Afrique, Paris, Cordier, 1830.

12. Voir R. Jamous, op. cit.

13. Tribu de la région de Tafraoute.

14. Tribu d'origine saharienne.

15. Tribu de la région d'Essaouira.

16. E. Gellner, Les saints de l'Atlas, Saint-Denis, Bouchène, 2003 (rééd.), p. 135-136, 144, 154, 235, 255,260 et 287.

17. E. Gellner, op. cit., p. 144. 
18. Terme sans nul doute dérivé de Bortugal (Portugal), même s'il est rare qu'il renvoie consciemment à l'État du Portugal. À ce propos, un jour, un de mes interlocuteurs me posa la question de savoir si les brtgz étaient des engliz!

19. Les archéologues de la délégation du patrimoine culturel de la région de Tiznit.

20. Capitaine Maitrot, "Les ruines dites portugaises des Doukkala », Archives berbères, vol. 1, fasc. 4, 1916, p. 279-292.

21. E. Gellner, op. cit., p. 255.

22. À ce sujet voir V. Monteil, "Chronique de la zaouia d'Assa ", in Mélanges Mohamed El-Fassi, Rabat, Université Mohamed-V, 1967, p. 81-90.

23. Op. cit., p. 171.

24. Dans la tradition coranique, Alexandre le Grand, appelé Seydna Duul Qarnain, est considéré comme un prophète.

25. F. Colonna, "Ce que les paysans disent de leurs ruines. Aurès, les années soixante-dix », Bulletin économique et social du Maroc, $\mathrm{n}^{\circ}$ 159-160-161, 1987, p. 249.

26. Traduction du terme tslim, qui vient de l'arabe salama, la paix, employé pour désigner l'acte de visiter et de prier le saint.

INDEX

Mots-clés : espace, histoire, frontière

Keywords : Southern Morocco, Ait Ba'amran, space, history, border

Index géographique : Sud marocain, Aït Ba'amran

\section{AUTEUR}

\section{ROMAIN SIMENEL}

Doctorant en ethnologie à l'université Paris 10-Nanterre, Romain Simenel est allocataire de recherche du ministère des affaires étrangères au Centre Jacques Berque à Rabat depuis 2003. Après deux années de recherche de terrain dans le Sud du Maroc, il termine la rédaction d'une thèse qui porte sur les régimes d'historicité et de spatialité chez les Aït Ba'amran, confédération tribale de la région de Sidi Ifni. Il est l'auteur de : « De la forêt du saint au pâturage des chrétiens. Perception du paysage et gestion du couvert végétal chez les Aït Ba'amran du Sud marocain », Cahiers de recherche du Centre Jacques Berque, I, 2004, p. 119-133.

ainrom@hotmail.com 